\title{
Imaging single bacterial cells with electro-optical impedance microscopy
}

Fenni Zhang ${ }^{\mathrm{a}}$, Shaopeng Wanga*, Yunze Yang ${ }^{\mathrm{a}}$, Jiapei Jiang,c, and Nongjian Tao ${ }^{\mathrm{a}, \mathrm{b}^{*}}$ \&

${ }^{a}$ Biodesign Center for Bioelectronics and Biosensors, Arizona State University, Tempe, Arizona 85287-5801, USA

b School of Electrical Computer and Energy Engineering, Arizona State University, Tempe, Arizona 85287, USA.

c School of Biological and Health Systems Engineering, Arizona State University, Tempe, Arizona 85287, USA.

*Corresponding authors:

Shaopeng Wang: shaopeng.wang@,asu.edu

Nongjian Tao: § Deceased in March 2020. 
S1. Linear relationship between optical responses and charge density with bare ITO

To explore the relationship between optical responses and charge density, we measured the optical response of bare ITO surface with voltage modulation at different frequencies. The bare ITO surface is modeled with Randles model by three electrical components, including solution resistance $\left(R_{S}\right)$, double layer capacitance $\left(C_{p}\right)$ arising from the ITO-solution interface and polarization resistance $\left(R_{c y t}\right)$. The ITO impedance $(Z)$ can be modelled by the following equation

$$
Z=R_{S}+\frac{1}{\frac{1}{R_{p}}+j 2 \pi f C_{p}}=\frac{V}{I}
$$

f is the modulation frequency. With the electric current recorded with different frequency modulation, we can calculate the impedance at the corresponding frequency and fit the result with Randles mode to extract the value of each parameter, as shown in Fig. S1A. With all the parameter extracted, the charge density of the ITO surface $\left(Q_{c}\right)$ can be calculated as

$$
Q_{c}=\frac{V R_{p} C_{p}}{R_{s}+R_{p}+j 2 \pi f R_{s} R_{p} C_{p}}
$$

Then, we compared the optical response (FFT amplitude) of the ITO surface with the calculated charge density. A linear relationship was observed, as shown in Fig. S1B. 

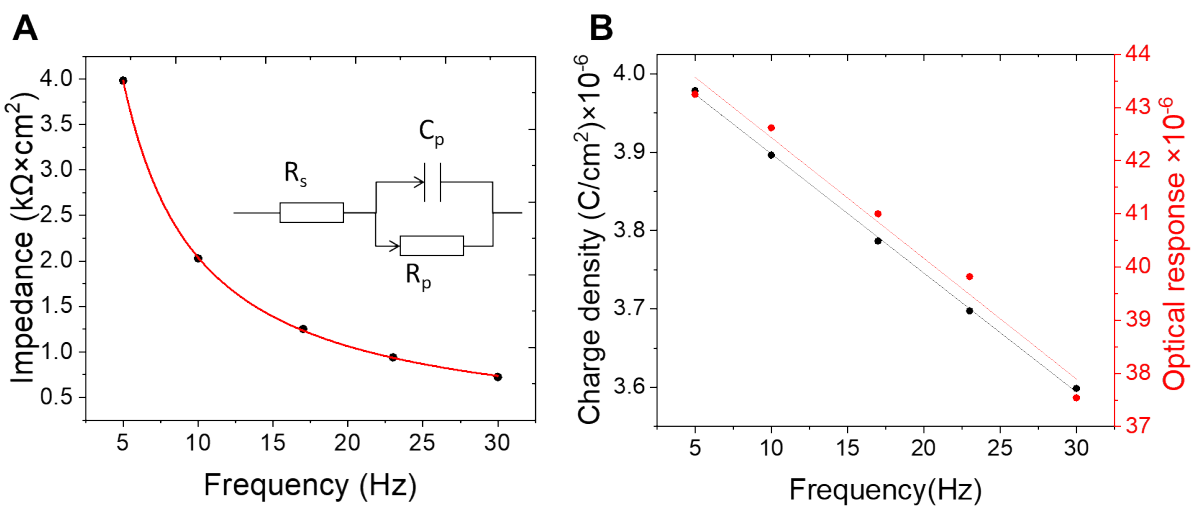

Fig. S1. The relationship between optical responses and charge density with bare ITO. (A) The calculated ITO impedance at different frequencies and the fitting model. (B) Linear relationship between calculated ITO charge density and the corresponding optical responses.

S2. Single cell tracking with imageJ
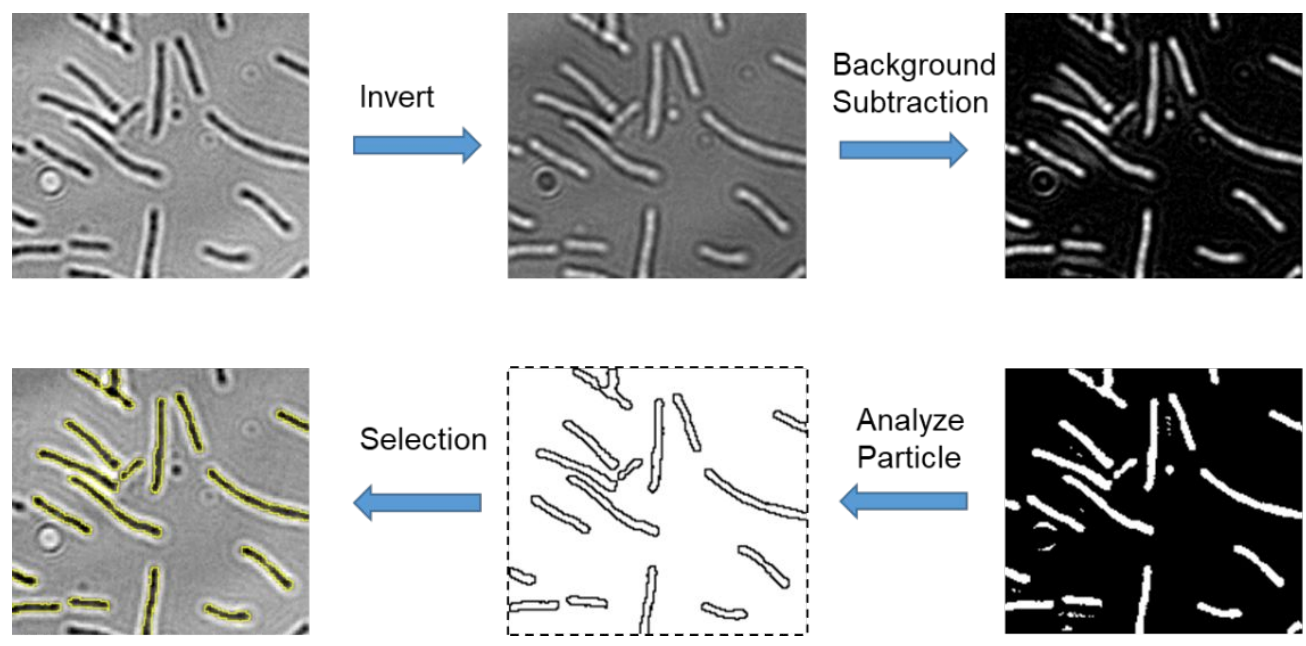

Fig. S2. Flow chart of single bacterial cell tracking with ImageJ

S3. Comparison of bacterial EIM response with ITO response 
A
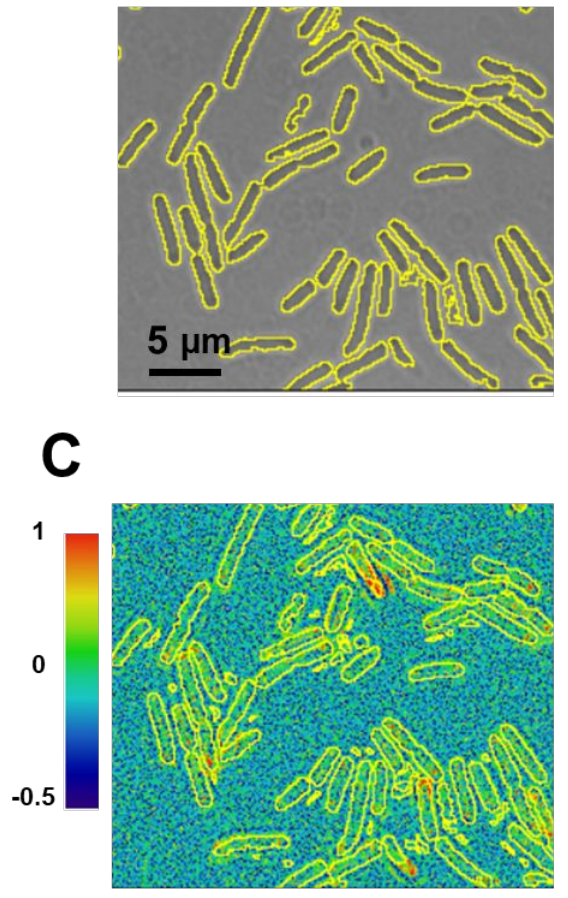

B
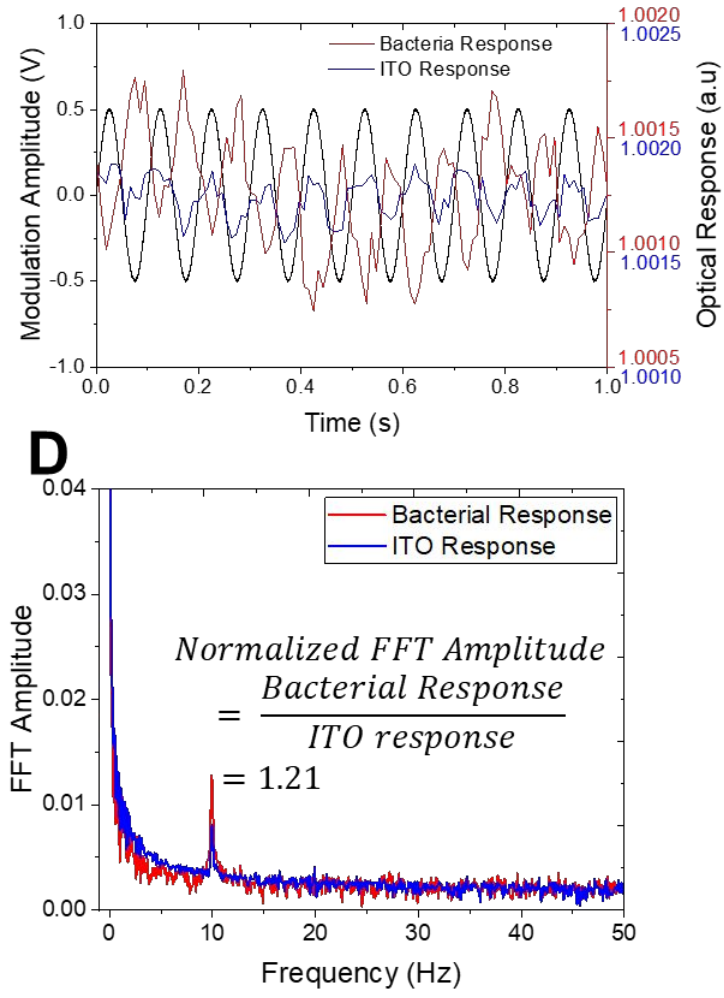

Fig. S3. The comparison of bacterial EIM response with ITO response. (A) Optical image of bacterial cells with individual cell selected. (B) The representative optical response of bacteria and ITO surface with $0.5 \mathrm{~V}, 10 \mathrm{~Hz}$ modulation over $1 \mathrm{~s}$. (C) The FFT mapping at $10 \mathrm{~Hz}$ with individual cell selected. (D) The averaged FFT amplitude of bacteria and ITO area and the calculation of normalized FFT amplitude.

S4. Simultaneous electrical current recording

When the voltage modulation was applied, the corresponding electrical current was recorded. During the antibiotic application, the recorded current didn't show any significant change over the 90 min test. It is because the electric current measures the averaged response of the whole system, 
and the bacteria contribution is small compared to the parallel ITO current with low cell coverage. In contrary, EIM is sensitive to individual cell impedance changes regardless of cell density.

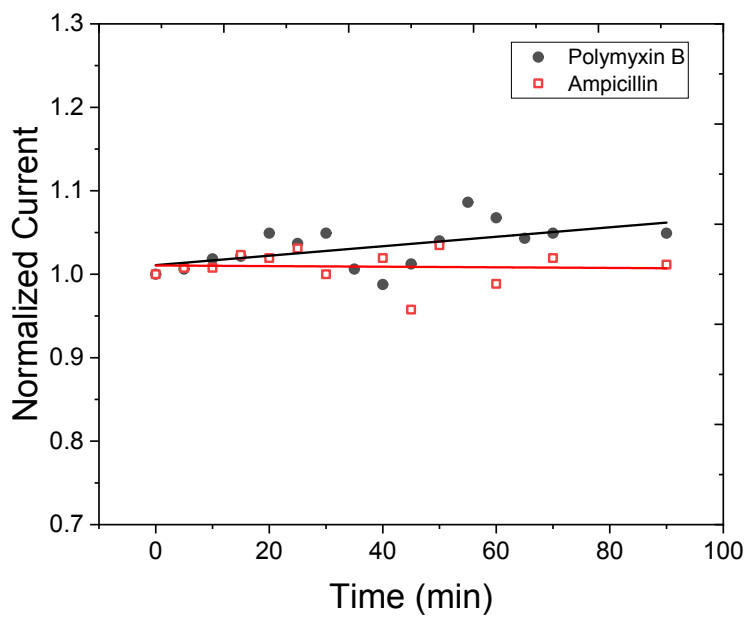

Fig. S4. The simultaneously recorded total electric current by the potentiostat during the EIM measurement of the bacteria responses to the two antibiotics. 